\title{
Second parameter globulars and dwarf spheroidals around the Local Group massive galaxies: What can they evidence?
}

\author{
V. Kravtsov` \\ Sternberg Astronomical Institute, University Avenue 13, 119899 Moscow, Russia \\ Received 29 April 2002 / Accepted 28 August 2002

\begin{abstract}
We suggest that the majority of the "young", so-called "second parameter" globular clusters (SPGCs) have originated in the outer Galactic halo due to a process other than a tidal disruption of the dwarf spheroidal (dSph) galaxies. Basic observational evidence regarding both the dSphs and the SPGCs, coupled with the latest data about a rather large relative number of such clusters among globulars in M 33 and their low portion in M 31, seems to be consistent with the suspected process. It might have taken place within the system of the most massive galaxies of the Local Group (LG) at the earliest stages of their formation and evolution. We argue that the origin and basic characteristics of the SPGCs can naturally be explained as a result of mass outflow from M 31, during and due to formation of its Pop. II stars, and subsequent accretion of gas onto its massive companions, the Galaxy and M 33. An amount of the gas accreted onto the Milky Way is expected to have been quite enough for the formation in the outer Galactic halo not only of the clusters under consideration but also a number of those dSph galaxies which are as young as the SPGCs. A less significant, but notable mass transfer from the starbursting protoGalaxy to the massive members of the LG might have occurred, too.
\end{abstract}

Key words. Galaxy: formation - Galaxy: halo - globular clusters: general - galaxies: dwarf - galaxies: interactions

\section{Introduction}

Color-magnitude diagram (CMD) studies of the Galactic globular clusters (GCs) accumulated in 1960-ies have convincingly shown that metallicity is not the only parameter governing the clusters' horizontal branch (HB) morphology and that some other parameter(s) affect the distribution of stars on the HB. In other words, a "second parameter" effect takes place: at a given metallicity, GCs with the second parameter phenomenon exhibit redder HB morphology. Thanks to numerous CMD studies, with limiting magnitude below the main sequence turnoff, of GCs in the Milky Way and in its nearest satellites, age has been revealed to play an important role in the second parameter effect. However, different investigators make contradictory conclusions regarding whether or not it is mainly responsible for the effect (for comparison, see Stetson et al. 1996 and Sarajedini et al. 1997; or VandenBerg 2000).

We stress the point that the second parameter problem itself is beyond the scope of the present paper. However, since the Galactic GCs under study have historically been called SPGCs, it is this term that is used hereafter to denote GCs younger than primeval ones, but with approximately the same mean metallicity, close to $[\mathrm{Fe} / \mathrm{H}] \sim-1.6$.

\footnotetext{
* e-mail: scorpi@sai.msu.ru
}

It is now well recognized (for instance, Zinn 1993; van den Bergh 1993; Lee et al. 1994) that GCs in the outer halo of the Milky Way form two subpopulations, "old" and "young". The latter consists of the SPGCs displaying predominantly red HB morphology. Presently, there is little doubt about the younger ages (by $\sim 2 \mathrm{Gyr}$ ) of the SPGCs as compared to the old GCs in the Galactic halo (e.g., Lee et al. 1994). In addition to such age characteristics, their predominant location in the halo at Galactocetric distance $R_{\mathrm{GC}}>8 \mathrm{kpc}$ poses the fundamental problem of the origin of these clusters. It is a longstanding, poorly understood problem that arose more than three decades ago. Revealing the cause of these GCs in the outer Galactic halo would be beneficial in understanding their formation and the early history of the Milky Way, and other related questions.

By proceeding from the tentative inference that age is responsible for the second parameter, Searle \& Zinn (1978), in their scenario of halo formation, hypothesized that the origin of SPGCs was in gaseous condensations, "transient protogalactic fragments that continued to fall into dynamical equilibrium with the Galaxy for some time after the collapse of its central regions had been completed". In this scenario the authors also considered the possibility of merging of low-mass galaxies with the Milky Way. Later, owning to development 
of the cold dark matter theories of galaxy formation, some investigators tentatively identified these hypothetical transient fragments with "dark halos" which, in turn, were found in the dSph galaxies, real objects with both the appropriate masses of visible matter and signs of a rather high dark matter content (see, among others, the review of various topics related to dSphs by Gallagher \& Wyse 1994; review by Mateo 1998). Moreover, a number of important observations - in particular, the discovery by Ibata et al. (1994) of the Sagittarius dSph that shows obvious signs of tidal disruption; findings about the second parameter effect displayed by the old stellar populations in $\mathrm{dSphs}$ and by GCs belonging to them (e.g., among others, Buonanno et al. 1985; Suntzeff et al. 1993; Demers \& Irwin 1993); a highly ordered spatial distribution of the Galactic dSph galaxies, as noted by Kunkel \& Demers (1976) and Lynden-Bell (1982), along with a close resemblance between three-dimentional distribution of SPGCs having the reddest HB morphology and some of the dSphs (Majewski 1994) - are evidence suggesting that the origin of the Galactic SPGCs is a consequence of tidal disruption/stripping (TD/S), during a Hubble time, of the formerly more numerous dSph-like systems or larger Galactic companion(s). Thus, the role of such a mechanism is at present widely believed to be crucial, and its reliability with regard to the formation of the Galactic halo is currently being investigated (for instance, Morrison et al. 2000; Harding et al. 2001).

Explaining the population of Galactic SPGCs by TD/S of the respective ancestral galaxies, $\mathrm{dSph}$ or some other satellites to the Milky Way appears to be a natural and well grounded approach to the problem. However, even if it was an absolute fact it would simply replace the problem by other one $(s)$ related to the origin of the ancestors themselves. Indeed, the Galactic SPGCs are, on the one hand, younger than the old halo globulars, but, on the other hand, they should have been among the oldest constituents of their hypothetical parent (dSph) systems. Therefore, we have to conclude that these tidally dissolved dSphs are younger (by $\sim 2$ Gyr or so) than the oldest objects populating the Galactic spheroid. Then, we need to explain the mysterious emergence of the "young" satellite galaxies in the outer Galactic halo, in about two Gyr after the beginning of its formation, as well as their relatively rapid subsequent dissolution by the Galactic tidal forces. Moreover, different facts and their detailed analysis (see Sect. 2) can militate against such an attractive "destructive" mechanism as being the major one responsible for the Galactic SPGC system formation.

Instead of (or in addition to) this we have recently suggested (Kravtsov 2001), and here we analyze in detail, a "creative" process which would perhaps be able to explain the origin of populations of both SPGCs and dSphs, belonging not only to the Galaxy but also to two other massive galaxies of the LG. Specifically, M 31 includes six dSph systems (Grebel 2000a), while M 33 has no dwarf companions, but according to data of Sarajedini et al. (1998) it has appreciable population of SPGCs.

The role of the $\mathrm{dSph}$-consuming process in the formation of the Galactic SPGCs population and populations of sush GCs in M 33 and M 31 is analyzed in the next section, taking into consideration diverse observational data obtained to date.
In Sect. 3, we summarize the basic characteristics of both the population of Galactic SPGCs and the dSph galaxies, which may be consistent with and explained by the processes described in Sect. 4.

\section{Is dSph-consuming process responsible for the origin of the SPGCs?}

To achieve a more adequate understanding of the nature of the Galactic SPGC population, observational information about such clusters in other massive galaxies of the LG is very valuable. Preliminary relevant results obtained to date seem to increase doubts about the TD/S of $\mathrm{dSphs}$ as the universal mechanism of and main contributor to the formation of populations of SPGCs in the three most massive galaxies of the LG. Contrary to expectations following from the large mass of M 31 and hence its comparatively high destructive ability, among GCs studied in the galaxy there are only hints of the existence of SPGCs (Rich et al. 2001). This means that the relative number of these clusters among GCs in M 31 is presumably low. Selection effects can take place though, taking into account that among Galactic globulars the SPGCs have, on average, lower concentrations and luminosities (van den Bergh 1996). At the same time, a surprisingly large number of GCs, eight out of ten in the sample studied by Sarajedini et al. (1998) in M 33, have turned out to show exclusively red HB morphology at the mean metallicity $[\mathrm{Fe} / \mathrm{H}]=-1.6$, i.e. they are the SPGCs. The authors carefully selected most probable candidates for the halo objects of M 33, and hence relative number of SPGCs in the halo of the Triangulum nebula is apparently high. This is further supported by the latest investigation by Chandar et al. (2002). They found that $\sim 60 \%$ of 17 kinematically selected halo clusters of M 33 appear to be "young" ones, "a much higher fraction of young to old halo clusters than found in the Milky Way". The observed clusters' projected distances from the galaxy center, at the given mass of the galaxy, do not exclude the origin of SPGCs in M 33 due to tidal disruption of hypothetical dSphs (but not to stripping, because we see no companions to M 33). However, the observations by Sarajedini et al. and by Chandar et al. (2002) coupled with recent result of Chandar et al. (2001) are able to put significant constraints on such a possibility. The latter authors have estimated the total number of GCs in M 33 to be $75 \pm 14$, which gives a specific frequency significantly higher than in other late-type spirals. It is most probable that this surprising excess is due to the presence of SPGCs. Therefore, in the framework of the dSphconsuming view of the origin of SPGCs we should again assume, as in the case of our Galaxy, the mysterious emergence of the hypothetical "young" satellite dSph galaxies in the halo of M 33 a few Gyr after the beginning of its formation. Moreover, it is necessary to accept a surprisingly complete disruption of all these dwarfs, as well as their preferably high mass, since only the high-mass dwarfs would be able to form GCs. These processes and characteristics seem to be fairly strange and artificial, and they could hardly be real in such a combination as applied to M 33. But, there is little doubt that some specific process(es) occurred in the halo of M 33 that led to 
both the prolonged formation of GCs and their obvious surplus population in the galaxy compared to those in many other latetype spiral galaxies.

Important constraints on the Galactic halo formation by the $\mathrm{dSph}$-consuming process come from the latest abundance investigations. Shetrone et al. (2001) have determined and analyzed abundance ratios for more than 20 elements in spectra, obtained with the Keck I telescope, of red giant stars belonging to the Draco, Sextans, and Ursa Minor dSphs. From a comparison of the measured values with published ones for the Galactic halo stars, a number of essential differences between them was found, particularly in $[\alpha / \mathrm{Fe}]$. Hence, the authors have concluded that their observations do not support the notion "that the Galactic halo has been assembled entirely through the disruption of very low-luminosity $\mathrm{dSph}$ galaxies like the three galaxies" studied by them. From a similar comparison of element abundance ratios measured for the Galactic halo stars and dSph stars within the same range of metallicity, Fulbright (2002) has made even stronger claims that the former stars in the solar neighborhood are unlikely to have been constituents of disrupted dSphs similar to the studied ones.

Fairly strong limits to the Galactic halo formation via dSph satellite merging have been set by Unavane et al. (1996) and Gilmore et al. (2000) as well. According to their estimates no more than some $10 \%$ of the halo could have formed due to this process over approximately the last $10 \mathrm{Gyr}$. The latter authors also conclude that "either the dwarfs merged before they formed stars, or they never formed".

Interesting supplementary constraints on the Galactic SPGCs were analyzed by van den Bergh (1996).

In addition to the above-mentioned, indirect studies of possible TD/S of the hypothetical dwarf galaxies in the past, direct investigations were carried out regarding the impact of the TD/S on mass loss from the dSph "survivors" really existing in the outer Galactic halo. Unfortunately, the respective results obtained to date for some $\mathrm{dSph}$ galaxies are very different, and they now cannot provide a decisive answer to the problem. Apart from the conventional process of the TD/S of the Sagittarius dSph galaxy, indications of an extratidal population around the Ursa Minor dSph are reported by MartinezDelgado et al. (2001). A substantial extratidal population from the Carina dSph galaxy has been found by Majewski et al. (2000), who have determined the rate of current mass loss from the galaxy to be surprisingly high, namely of order of $27 \%$ of its mass every Gyr. Hence, they evaluate, under some assumptions, that $14 \mathrm{Gyr}$ ago the Carina $\mathrm{dSph}$ was 100 times more massive than now. At the same time, Odenkirchen et al. (2001) and Aparicio et al. (2001) have found no tidal tails or an extratidal extension of the stellar populations belonging to the Draco dSph galaxy. For comparison with some of the above findings it is interesting to note the latest results obtained by Hayashi et al. (2002), modeling the dark matter halos surrounding dSph satellites of the Milky Way. They find that these halos have circular velocity curves with peaks at essentially higher velocities than determined from the stellar velocity dispersion, and that the tidal radii of the $\mathrm{dSphs}$ are larger than those derived from observations.
Taken at face value, these observations may imply that a role played by the $\mathrm{dSph}$-consuming process in the formation of the population of SPGCs in the outer Galaxy, as well as populations of these clusters around the LG massive spirals, is secondary. For this reason, we suggest other process as being important. In our approach we proceed from the recognized starting point that there is an apparent relationship between the dSphs and SPGCs. However, we assume that both kinds of objects originated from the same cause rather than the dSphconsuming process resulted in the populations of SPGCs.

\section{Summary of the basic characteristics}

The relationship between the Galactic populations of dSphs and SPGCs is reliably shown by their basic characteristics which, in turn, should be consistent with the process suspected to be responsible for the origin of these objects. These characteristics are summarized below;

- The age of the SPGCs, as mentioned above, differs from that of the old GCs by a few Gyr, typically 2 Gyr (but see age data for some "young" globulars in VandenBerg 2000);

- These clusters exhibit peculiar kinematic characrestics. They have, on average, marginally retrograde or zero rotation, whereas old GCs show direct motion with lower dispersion. According to Da Costa (1994) the mean velocities of rotation are $V_{\text {rot }}=-45 \pm 81 \mathrm{~km} \mathrm{~s}^{-1}$ and $V_{\text {rot }}=58 \pm 24 \mathrm{~km} \mathrm{~s}^{-1}$, respectively;

- Metallicities of the SPGCs, as noted by Rodgers \& Paltoglou (1984) and confirmed by van den Bergh (1993), fall in a quite narrow range, within $\Delta[\mathrm{Fe} / \mathrm{H}]=0.4 \mathrm{dex}$, with no relation between metallicity and Galactocentric distance. However, Yoon \& Lee (2002) argue that the majority of the most metal-poor $([\mathrm{Fe} / \mathrm{H}]<-2.0)$ Galactic GCs are younger, by $\sim 1 \mathrm{Gyr}$, than the oldest globulars, and find that these clusters "display a planar alignment in the outer halo", close to the Magellanic Plane;

- Majewski (1994) has shown that three-dimentional distribution of the SPGCs with reddest HB morphology is such that they appear to populate the so-called "Fornax-Leo-Sculptor stream" consisting of five dSph galaxies orbiting the Milky Way. He has argued that all these objects "may be related through origin in a common Galactic accretion event";

- Moreover, Galactic dSphs themselves show ordered distribution on the sky, with the plane formed by them being nearly orthogonal to the Galactic plane (Kunkel \& Demers 1976; Lynden-Bell 1982; Majewski 1994). A similar ordered distribution is also displayed by dSphs belonging to the Andromeda galaxy. Both dSph populations around the two parent galaxies apear to be distributed in space either roughly near the same plane or near two planes with a low inclination between them, as may be seen, for example, in the results of Hartwick (2000) or in the Figs. 1 and 3 from Grebel (2000b) and Mateo (1998), respectively;

- In contrast to dwarf irregular galaxies, which are more or less uniformly distributed across the LG, dSphs show obvious concentration to the Galaxy and M 31 (e.g., Einasto et al. 1974; van den Bergh 1994; Grebel 2000a);

- CMDs of dSphs and their GCs, like those of the SPGCs, are strongly affected by the second parameter. This may 
be evidence that ages of at least some of the objects are younger than the old globulars. For instance, according to Suntzeff et al. (1993) the Sextans dSph galaxy "may be a few Gyr younger than the typical Galactic globular cluster". Saragedini et al. (2002) conclude that the Cetus dSph is 2-3 Gyr younger than Galactic GCs with the same metallicity, provided that age is primarily responsible for the second parameter. On the contrary, Buonanno et al. (1998) find no significant difference in age between GCs belonging to the Fornax dwarf galaxy and Galactic GCs M 68 and M 92. Also, the Ursa Minor dSph galaxy is estimated to be as old as the latter globular (Mighell \& Burke 1999). It should be noted here that, due to a number of well known reasons, the accuracy of the relative age estimates for the Galactic dSph galaxies is hardly better than $1 \mathrm{Gyr}$, even at best. In any case, it is quite probable that at least a number of the presently observed dSphs belonging to the Milky Way are not the primeval objects in the sense that their oldest stellar populations are younger than the old Galactic globulars. One can suggest that either such dSphs were formed in relation to some secondary process(es) or initial starbursts in the protodSphs ("dark halos") were delayed until they were triggered by these processes;

- Note also that both the number of dSphs orbiting the Galaxy and the total mass of luminous matter that they comprise, as one can deduce from the compilative data of Grebel (2000a), are apparently larger when compared to dSph systems belonging to M 31 .

\section{Putative consequence of the mass outflows}

It has been argued since 1970s (e.g., Larson 1974; Bookbinder et al. 1980; Marochnik \& Suchkov 1984; Berman \& Suchkov 1991) that some properties of galaxies, particularly chemical ones, and those of hot gas in clusters of galaxies imply a considerable mass loss which may have occurred in the early epoch of formation and evolution of the galaxies. This is mainly due to the mass outflows caused by supernovae-driven winds during the most powerfull (initial) star formation event(s). Such a loss of gas by galaxies such as M 31 and the Milky Way has been evaluated to amount up to a half mass of the respective protogalaxy, i.e. $\Delta M \sim 10^{11} M_{\odot}$.

These conclusions, reached in earlier studies, seem to be in general agreement with the latest results achieved on the basis of more advanced observational and theoretical investigations of starbursting galaxies at different redshift. The high-redshift Lyman break galaxies (LBGs) are suggested to be progenitors of the present-day massive spheroids being, in particular, components of luminous early-type spirals (Friaca \& Terlevich 1999, for instance). Starburst-driven galactic superwinds observed in LBGs play important role in the mass outflow from them and in enrichment of the intergalactic medium. As summarized by Heckman (2001, and references therein), "the outflows carry mass out of the starburst at a rate comparable to the star-formation rate". This implies that outflows from a starforming protogalaxy, like M 31, during the most powerful episode(s) of its Population II formation, that is (are) assumed to last $\sim 2 \mathrm{Gyr}$ with the star formation rate $\sim 100 M_{\odot} \mathrm{yr}^{-1}$ (Granato et al. 2001) would be able to carry off the protogalaxy a total mass of gas somewhat exceeding $\sim 10^{11} M_{\odot}$. Because according to Pettini et al. (2001) typical speeds of such a gas in LBGs range from a few $10^{2}$ to $10^{3} \mathrm{~km} \mathrm{~s}^{-1}$, some tens of percent of this mass, i.e. up to $\Delta M \sim 10^{11} M_{\odot}$ (that is in agreement with the former estimate), with velocities exceeding the escape velocity of M 31, could have left the galaxy.

If so, then portion of the gas that escaped from M 31 could be accreted onto its massive companion(s), our Galaxy (and M 33), as it might be expected from the well-known processes taking place in binary star systems. We can easily estimate an order of magnitude of a time delay between starting an expansion of the gas from M 31 and its subsequent accretion onto the Galaxy. If we build upon both the present distance between the two galaxies $(\sim 0.7 \mathrm{Mpc})$ and the ratio of their mass $\left(M_{\mathrm{G}} / M_{\mathrm{A}} \sim 1 / 1.5\right)$ then we find that the time spent by the gas to reach a region of the Lagrangian point between the two galaxies $(\sim 0.4 \mathrm{Mpc})$ may be as long as $1 \mathrm{Gyr}$ at a mean velocity of the expanding gas of the order of $\sim 400 \mathrm{~km} \mathrm{~s}^{-1}$. The assumed duration ( 2 Gyr) of mass loss from M 31 combined with the above time delay can explain, in particular, the mean age difference between the SPGCs and primeval globulars in our Galaxy.

Yet one key quantity is an upper limit of mass of the gaseous material $\delta M$ that might have been accreted onto the Galaxy if the total amount of gas lost by M 31 was $\Delta M\left(\sim 10^{11} M_{\odot}\right)$. For this purpose we have applied a formula deduced by Postnov (2000) in the approach of gas accretion in gravity potential $\varphi \sim \frac{1}{R}$ of the field of the less massive companion in a binary system. By substituting the corresponding values in the formula, we obtain:

$\delta M \leq \frac{1}{4}\left(\frac{M_{\mathrm{G}}}{M_{\mathrm{A}}}\right)^{2} \Delta M \sim \frac{1}{10} \Delta M \sim 10^{10} M_{\odot}$.

Even if a mass of really accreted gas was an order of magnitude lower than the upper limit estimated and if, in turn, approximately ten percent of this mass was converted into objects, i.e., $\sim 10^{8} M_{\odot}$, that would be equal to a total mass of the bulk of SPGCs, a few dSph galaxies, and also of field stars with their total mass 1-1.5 order of magnitude higher compared to the mass of all the SPGCs, which might have formed due to such a process in the outer halo of our Galaxy. The typical cluster and dSph masses are accepted to be $\sim 10^{5} M_{\odot}$ and $\sim 10^{7} M_{\odot}$, respectively. It is worth of noting that from a detailed analysis of the space motions of the Galactic halo stars, Chiba \& Beers (2000) find both an excess number of high-eccentricity stars and a sharp discontinuity, near zero, of the mean rotational velocities to occur near the same metallicity, $[\mathrm{Fe} / \mathrm{H}] \sim-1.7$. They suggest that a significant fraction of stars with metallicity near this value might form from infalling gas with the corresponding chemical abundance during the early formation of the Galaxy. Intriguingly enough, average metallicities of SPGCs in the Galaxy and $\mathrm{M} 33$, falling near $[\mathrm{Fe} / \mathrm{H}] \sim-1.6$, are very close to the above value.

Similarly, by applying the above formula to the case of M 33 and taking into account that its mass is a factor of ten lower than the mass of M 31, it is possible to conclude that objects assumed to form from a gas accreted onto M 33 might 
include some dozen (or more) GCs and a population of field stars too.

Apart from M 31, the early Galaxy is also expected to lose a sizable amount of partially enriched gas, though its overall mass should apparently be less than in the case of the former galaxy. Therefore, a less significant mass transfer, from the star-forming protoGalaxy to its massive companions, along with the respective consequences are suspected to have taken place too. Here, it is worth of mentioning that, by analyzing potometric properties of the paired early-type galaxies, Demin (1984) assumed the possibility of mass-exchange processes between such galaxies at the early stage of their evolution, and Demin \& Sazhin (1985a, 1985b) studied this possibility. In particular, in the latter paper the authors conclude that mass exchange between companions in a binary galactic system can be more preferable than a mass loss from the system.

Of course, we here use simple evaluations which are preliminary. However, they appear to be sufficient to show, in principle, the expected scale and consequences of mass transfer between the most massive members of the LG. Additionally, the numerous observations of present-day formation of massive star clusters and dwarf galaxies in the tidal tails of interacting galaxies (e.g., Gallagher et al. 2001 and references therein) may be important indirect observational evidence supporting the formation of such objects due to the suggested type of interaction between the LG massive galaxies during formation of their spheroids. At this stage of their formation, a high portion of mass of each of the starbursting galaxy remains in the form of a gas, part of which is contracting to the central regions of the spheroids, and another part is expanding out of them. It is gas in the outer regions of the star-forming protoGalaxy that could primarily collide with an accelerated infalling stream of gas which was being transferred from the Andromeda protogalaxy through a region of the Lagrangian point between the early galaxies. Supersonic gas motion and accretion, under a speed of the gaseous material relative motion reaching at least a few $10^{2} \mathrm{~km} \mathrm{~s}^{-1}$, should have led to shockwaves. Indeed, sound speed in gas with $T \approx 10^{6} \mathrm{~K}$ is near $150 \mathrm{~km} \mathrm{~s}^{-1}$, whereas the speed of the accelerated infalling gas might be not less than $200 \div 300 \mathrm{~km} \mathrm{~s}^{-1}$. Shockwaves are known to be powerful factor triggering and essentially increasing efficiency of star formation. Perhaps, the conditions favourable for the formation of stars and clusters were more or less met at a distance of several tens of kiloparsecs from the Galactic centre. The efficiency of star formation would probably be highest, irrespective of Galactocetric distance, if it happened that the infalling stream collided with relatively dense gas contained in the "dark halos". It is important to empasize that, by analogy with a mass transfer between companions in an interacting binary star system, the passage of gas through the Lagrangian point between spheroids of the two protogalaxies should have led the infalling gas to become denser and to have more or less ordered distribution and motion around the protoGalaxy, close to the plane defined by the relative motion of M 31 and the Milky Way at that moment. Therefore, the objects that could form from the infalling gas, thanks to the mass transfer from M 31 to the Galaxy, would exhibit signs of the respective kinematics and spatial distribution around the latter galaxy in its outer parts. The same should be correct for gas accretion onto M 33. The observed characteristics of the aforementioned stars with metallicity around $[\mathrm{Fe} / \mathrm{H}] \sim-1.7$ and populations of both the Galactic SPGCs and dSphs seem to agree well with the objects' origin due to such a process. Kinematic and spatial characteristics of SPGCs in M 33 also suggest their accretive origin in the halo of the galaxy (Chandar et al. 2002).

Similarly, it is not excluded that the infalling gas might have collided with the interstellar matter in the outer parts of the early LMC, the largest Galactic satellite. This could result in the induced formation of additional globulars more weakly bound to the LMC, which were subsequently captured by the Galaxy.

Accepting the proposed and discussed interaction between the Milky Way, the Andromeda galaxy, and the Triangulum nebula in their early history, we can naturally explain (in the present section) the existence and the basic characteristics (Sect. 3) of SPGCs and (at least) some dSphs orbiting these galaxies. We omit the problem dealing with the relationship between the number of SPGCs and field stars formed in the same star-forming process. It is related to the more general, complex problem of GC (massive cluster) formation and its efficiency (e.g., Larsen \& Richtler 2000; Elmegreen 2001). Note only that for the populations of "normal", metal-poor and metal-rich GCs belonging to either elliptical galaxies or spheroids of spirals such a relationship may vary essentially (e.g., Harris et al. 1999 and Durrell et al. 2001).

Observations of high redshift structures of galaxies, such as reported by Venemans et al. (2002), would perhaps be able to shed light on the problem of the mass transfer between massive galaxies at early stage of their evolution.

\section{Conclusions}

The present paper discusses long-standing problem of the origin of the Galactic SPGCs. We show that there arise questions about the currently popular and conventional view on the origin of a population of these globulars as a result of TD/S of dSph galaxies, especially taking into account recent relevant observational data including those on the populations of such globulars in the two other most massive galaxies of the LG, M 31 and M 33. On the one hand, answering these questions requires a number of a priori assumptions, some of which seem to be rather artificial and contradictory, about hypothetical events and objects. On the other hand, we argue that even if this view is correct, the problem is merely replaced by other one(s) related to the origin of $\mathrm{dSphs}$ themselves. In order to interpret and reconcile the various observational data on SPGCs (and probably dSphs) belonging to the three spirals of the LG we propose here other scenario. It is based on the realistic suggestion about natural consequences of mass loss from M 31 in its early history, namely, subsequent partial accretion of the gaseous material onto the early Galaxy and M 33 should have contributed to the formation of their (outer) halos.

In addition, we draw attention to the possible interaction between massive galaxies at the early stage of their formation and evolution in pairs, groups, and perhaps in small clusters. In contrast to the relatively well-known and recognized 
consequences of a tidal interaction between galaxies, the suspected role of the interaction we deal with seems to be presently missing or underestimated. We suggest that if some starbursting massive protogalaxy, like M 31 or our Galaxy, really loses a substantial amount of its gas, then the partially enriched gas expanding out and escaping from the protogalaxy not only contributes to the intergalactic medium but also should partially be trasferred to massive companion(s) of the protogalaxy. Because of the sizable amount of the gaseous material (probably, around a few per cent of mass lost by the starbursting massive protogalaxy) trasferred to and accreted onto the massive companions, they may experience additional large-scale starbursts leading to the formation, out of this material, of supplementary objects (stars, star clusters, and even dwarf galaxies) with particular appropriate characteristics. Therefore, strictly speaking, such galaxies do not form and evolve as completely isolated entities, and they may contain populations of SPGCs (and probably dSphs) as tracers of the interaction under study.

We suppose that just this kind of interaction occurred between the most massive galaxies of the LG and lasted approximately 2-3 Gyr after the beginning epoch of their formation, and it could primarily be responsible for the origin of both their SPGC populations and some of the dSph galaxies, as well as for the basic observables of these objects.

Acknowledgements. The author is very grateful to A. A. Suchkov for his first encouraging comments on the idea of the scenario presented, and to $\mathrm{S}$. van den Bergh whose attention and interest in the scenario outline presented in astro-ph was important moral support. Thanks are due to Yu. N. Efremov, K. Postnov, A. S. Rastorguev, M. V. Sazhin, N. I. Shakura, Yu. A. Shchekinov, and V. V. Slavutinsky for useful discussions. L. Murray's assistance in improving the English of the paper is acknowledged. The author thanks an anonymous referee whose useful criticism stimulated improvement of the paper.

\section{References}

Aparicio, A., Carrera, R., \& Martinez-Delgado, D. 2001, AJ, 122, 2524

Berman, B. G., \& Suchkov, A. A. 1991, Ap\&SS, 184, 169

Bookbinder, J., Cowie, L. L., Krolik, J. H., Ostriker, J. P., \& Rees, M. 1980, ApJ, 237, 647

Buonanno, R., Corsi, C. E., Fusi Pecci, F., Hardy, E., \& Zinn, R. 1985, A\&A, 152, 65

Buonanno, R., Corsi, C. E., Zinn, R., et al. 1998, ApJ, 501, L33

Chandar, R., Bianchi, L., \& Ford, H. C. 2001, A\&A, 366, 498

Chandar, R., Bianchi, L., Ford, H. C., \& Sarajedini, A. 2002, ApJ, 564, 712

Chiba, M., \& Beers, T. C. 2000, AJ, 119, 2843

Da Costa, G. S. 1994, in ESO Conference and Workshop Proceedings 51, The Local Group: Comparative and Global Properties, ed. A. Layden, R. C. Smith, \& J. Storm, 101

Demers, S., \& Irwin, M. J. 1993, MNRAS, 261, 657

Demin, V. V. 1984, SvA, 28, 622

Demin, V. V., \& Sazhin, M. V. 1985, Moskovskii Universitet, Vestnik, Seriia 3 - Fizika, Astronomiia, 26, (2), 100

Demin, V. V., \& Sazhin, M. V. 1985, Moskovskii Universitet, Vestnik, Seriia 3 - Fizika, Astronomiia, 26, (3), 74

Durrell, P. R., Harris, W. E., \& Pritchet, C. J. 2001, AJ, 121, 2557
Einasto, J., Saar, E., Kaasik, A., \& Chernin, A. D. 1974, Nature, 252, 111

Elmegreen, B. G. 2001, in Extragalactic Star Clusters, ed. E. K. Grebel, D. Geisler, \& D. Minniti, IAU Symp. Ser., 207 [astro-ph/0110147]

Friaca, A. C. S., \& Terlevich, R. J. 1999, MNRAS, 305, 90

Fulbright, J. P. 2002, AJ, 123, 404

Gallagher, J. S., \& Wyse, R. F. G. 1994, PASP, 106, 1225

Gallagher, S. C., Charlton, J. C., Hunsberger, S. D., Zaritsky, D., \& Whitmore, B. C. 2001, AJ, 122, 163

Gilmore G., Hernadez, X., \& Valls-Gabaud, D. 2000, in Proc. of the 35th Liege International Astrophysics Colloq., The Galactic Halo: From Globular Cluster to Field Stars, ed. A. Noels, P. Magain, D. Caro, et al., 325

Granato, G. L., Silva, L., Monaco, P., et al. 2001, MNRAS, 324, 757

Grebel, E. K. 2000a, in Proc. of the 33rd ESLAB Symp., ESA SP-445, Star Formation from the Small to the Large Scale, ed. F. Favata, A. A. Kaas, \& A. Wilson, 87 [astro-ph/0005296]

Grebel, E. K. 2000b, in ASP Conf. Ser., Microlensing 2000: A New Era of Microlensing Astrophysics, ed. J. W. Menzies, \& P. D. Sackett [astro-ph/0008249]

Harding, P., Morrison, H. L., Olszewski, E. W., et al. 2001, AJ, 122, 1397

Harris, G. L. P., Harris, W. E., \& Poole, G. B. 1999, AJ, 117, 855

Hartwick, F. D. A. 2000, AJ, 119, 2248

Hayashi, E., Navarro, J. F., Taylor, J. E., Stadel, J., \& Quinn, T. 2002, ApJ, submitted [astro-ph/0203004]

Heckman, T. M. 2001, In ASP Conf. Proc., 240, Gas and Galaxy Evolution, ed. J. E. Hibbard, M. Rupen, \& J. H. van Gorkom, 345 [astro-ph/0009075]

Ibata, R. A, Gilmore, G., \& Irwin, M. J. 1994, Nature, 370, 1941

Kravtsov, V. V. 2001, in Extragalactic Star Clusters, ed. E. K. Grebel, D. Geisler, \& D. Minniti, IAU Symp. Ser., 207 [astro-ph/0106227]

Kunkel, W. E., \& Demers, S. 1976, Roy. Green. Obs. Bull., 182, 241

Larsen, S. S., \& Richtler, T. 2000, A\&A, 354, 836

Larson, R. B. 1974, MNRAS, 169, 229

Lee, Y.-W., Demarque, P., \& Zinn, R. 1994, ApJ, 423, 248

Lynden-Bell, D. 1982, Observatory, 102, 202

Majewski, S. R. 1994, ApJ, 431, L17

Majewski, S. R., Ostheimer, J. C., Patterson, R. J., et al. 2000, AJ, 119, 760

Marochnik, L. S., \& Suchkov, A. A. 1984, Galaktika (Nauka Publ., Moscow, book in Russian)

Martinez-Delgado, D., Alonso-Garcia, J., Aparicio, A., \& Gomez-Flechoso, M. A. 2001, ApJ, 549, L63

Mateo, M. 1998, ARA\&A, 36, 435

Mighell, K. J., \& Burke, C. J. 1999, AJ, 118, 366

Morrison, H. L., Mateo, M., Olszewski, E. W., et al. 2000, AJ, 2000, 119,2254

Odenkirchen, M., Grebel, E. K., Harbeck, D., et al. 2001, AJ, 122, 2538

Pettini, M., Steidel, C. C., Shapley, A. E., et al. 2001, in Proc. of the ESO/ECF/STScI Workshop, Deep Fields, ed. S. Cristiani, A. Renzini, \& R. E. Williams (Springer), 35 [astro-ph/0101254]

Postnov, K. 2000, private communication

Rich, R. M., Corsi, C. E., Bellazzini, M., et al. 2001, in Extragalactic Star Clusters, ed. E. K. Grebel, D. Geisler, \& D. Minniti, IAU Symp., 207, [astro-ph/0106015]

Rodgers, A. W., \& Paltoglou, G. 1984, ApJ, 283, L5

Sarajedini, A., Chaboyer, B., \& Demarque, P. 1997, PASP, 109, 1321

Sarajedini, A., Geisler, D., Harding, P., \& Schommer, R. 1998, ApJ, 508, L37 
Sarajedini, A., Grebel, E. K., Dolphin, A. E., et al. 2002, ApJ, accepted [astro-ph/0111365]

Searle, L., \& Zinn, R. 1978, ApJ, 225, 357

Shetrone, M. D., Cote, P., \& Sargent, W. L. W. 2001, ApJ, 548, 592

Stetson, P. B., Vandenberg, D. A., \& Bolte, M. 1996, PASP, 108, 560 Suntzeff, N. B., Mateo, M., Terndrup, D. M., et al. 1993, ApJ, 418, 208

Unavane, M., Wyse, R. F. G., \& Gilmore, G. 1996, MNRAS, 278, 727

VandenBerg, D. A. 2000, ApJS, 129, 315

van den Bergh, S. 1993, ApJ, 411, 178 van den Bergh, S. 1994, in ESO Conf. and Workshop Proc. 51, The Local Group: Comparative and Global Properties, ed. A. Layden, R. C. Smith, \& J. Storm, 3

van den Bergh, S. 1996, PASP, 108, 986

Venemans, B. P., Kurk, J. D., Miley, G. K., et al. 2002, ApJ, accepted [astro-ph/0203249]

Yoon, S.-J., \& Lee, Y.-W. 2002, Science, 297, 578

Zinn, R. 1993, in The Globular Cluster-Galaxy Connection, ed. G. H. Smith, \& J. P. Brodie, ASP Conf. Ser., 48, 38 